\title{
Protective effects of Atractylenolide III on inflammation and oxidative stress in ovalbumin-induced asthma mice and its possible mechanisms
}

\author{
Lizhi Zhang ${ }^{1, *}$, Hengzhong $\mathrm{Yi}^{2, *}$, Di Jiang ${ }^{3}$ and Zongping $\mathrm{Li}^{4}$ \\ ${ }^{1}$ Orthopedics and Integration Medical Department, Hunan Chest Hospital, Changsha, Hunan, China \\ ${ }^{2}$ 6th Medical Department, Hunan Chest Hospital, Changsha, Hunan, China \\ ${ }^{3}$ Department of Ultrasound, the First Affiliated Hospital of University of South China, Hengyang, Hunan, China \\ ${ }^{4}$ Department of Geriatrics, Taizhou Hospital of Linhai City, Zhejiang, China
}

\begin{abstract}
Asthma is a complex disorder characterized by chronic inflammation of the airways. We aimed to investigate the role of Atractylenolide III (ATL III) in ovalbumin (OVA)-induced mouse asthma. Asthma was induced to BALB/c mice by sensitization with intraperitoneal injection of OVA, followed by treatment with ATL III. Pathological changes in lung tissue were examined by hematoxylin/eosin and sirius red staining. The levels of inflammation- and oxidative stress-related factors in the bronchoalveolar lavage fluid (BALF) were monitored using kits. Additionally, the contents of inflammatory cells including macrophages, lymphocytes, eosinophils and neutrophils in BALF were counted. The expression of signal transducer and activator of transcription 3 (STAT3) was tested using Western blotting and immunohistochemistry assay. Results revealed that ATL III markedly attenuated OVA-induced pathological injury of lung tissues in mice. Furthermore, ATL III controlled the cytokines production and balanced the oxidative stress condition, which was exhibited by the reduced levels of inflammation- and oxidative stress-related factors. Moreover, mice in ATL III-treated groups presented less inflammatory cells in BALF and ATL III largely inhibited STAT3 expression in lung tissues. Taken together, ATL III alleviates inflammation, oxidative stress and is associated with changes in pulmonary functions in a mouse asthma model through inhibiting STAT3.
\end{abstract}

Key words: Asthma — Atractylenolide III — Inflammation — Oxidative stress — STAT3

\section{Introduction}

Asthma is a chronic inflammatory disease characterized by mucus hypersecretion, airway hyperresponsiveness and airway wall remodeling that results in variable airway obstruction in susceptible individuals (Edwan et al. 2004; Dogan et al. 2020). It is estimated that asthma causes about 250,000 premature deaths annually, and that more than 300 million people are affected by it globally every year according to the World Health Organization (WHO). And this number is expected to rise to 400 million by 2025 (Beharry et al. 2015; Loftus and Wise 2016). It is crucial to develop a standard

\footnotetext{
* These authors contributed equally to this work.

Correspondence to: Zongping Li, Taizhou Hospital, 150 Ximen Street, Gucheng Street, Linhai, Zhejiang, 317000, China E-mail: ql123hyac@126.com
}

therapy for asthma, as it is a leading cause of disability, poor quality of life and increased cost of public disease management (Corrado et al. 2013; Loftus and Wise 2015).

Dysfunction of immune cells plays a crucial role in the progression of asthma. A growing body of literature has shown that imbalances in T helper 1 (Th1)/Th2 and Th17/ Treg may be the core pathogenesis of allergic asthma (Ji et al. 2014; Liang et al. 2017). Th1 cells release cytokines such as interferon- $\gamma$ (IFN- $\gamma$ ), interleukin (IL)-10, IL-12. Th 2 cells release IL-4, IL-5 and IL-13, leading to the accumulation of eosinophils (EOS) in the lungs of asthmatic patients, which is essential for the development of asthma (Foster et al. 2001). Inflammatory immune cells, including neutrophils (NEU), macrophages (MAC), lymphocytes (LYM) and EOS, increase the levels of reactive oxygen species (ROS) to exacerbate inflammation and injury in lung tissue (de Groot et al. 2019). Several signaling pathways have been 
described to be engaged in asthma physiopathology. The signal transducer and activator of transcription (STAT) are a family of cytokine and growth factor-inducible transcription factors that are critical in immune and inflammatory responses, which can be activated under inflammation condition (Hodge et al. 2005). Accumulating evidence shows that the expression of STAT3 is activated in ovalbumin (OVA)-induced allergic inflammatory response, which plays a significant role in the development of asthma (Huang et al. 2018). Cytokines from Th2 cells driven by STAT3 are believed to play an active role in establishing the chronic inflammation of asthma (Lim et al. 2015). Studies have proven that suppression of STAT3 gene expression is a therapeutic approach to asthma treatment (SimeonePenney et al. 2007; Nikolskii et al. 2019).

Despite substantial progress in asthma management, new asthma interventions are still needed as contemporary approaches have their limitations (Jones 1989; Sarver and Murphy 2009). Atractylenolide III (ATL III), the major bioactive component of Atractylodes macrocephala Koidz, has been demonstrated to possess anti-oxidative, anti-bacterial, anti-allergic response and anti-tumour properties (Zhang et al. 2013; Zhao et al. 2015). It has been substantiated that this sesquiterpene lactone relieves cerebral ischemic injury and neuroinflammation through inhibition of JAK2/STAT3/ Drp1-dependent mitochondrial fission (Zhou et al. 2019). In addition, compelling evidence indicates that ALT III attenuates inflammation by restraining the activation of NF- $\kappa B$ and reducing the secretion of TNF- $\alpha$ and IL-6 (Li et al. 2007; Kang et al. 2011; Ji et al. 2016). In parallel, ALT III is reported to inhibit the expression of STAT3 (Zhou et al. 2019). However, the effects of ALT III in asthma are not yet documented. Based on the above research findings, the current study aimed to investigate whether ALT III can suppress the allergic inflammatory response in an OVAsensitized mouse asthma model through the inhibition of STAT3 expression.

\section{Materials and Methods}

\section{Animals}

Fifty-four 12-week-old female BALB/c mice (18-22 g) were purchased from the Model Animal Center of Nanjing University (Nanjing, China). Animals were maintained in SPF level environment under a 12 -h light/dark cycle at $21 \pm 3^{\circ} \mathrm{C}$. Mice had free access to water and regular diet. They were housed for at least one week before the experimental performance. The animal protocols used in this study were approved by the Ethics Committee on Animal Experiments of the First Affiliated Hospital of University of South China. And all efforts were made to minimize the suffering of mice.

\section{Establishment of OVA mice model and drug treatment}

OVA-induced mouse model was developed in the above mentioned BALB/c mice by adopting the previous protocol (Liang et al. 2017). Briefly, mice were immunized with $100 \mu \mathrm{g}$ OVA (A5503, Grade V; Sigma-Aldrich, St. Louis, MO, USA) emulsified in $1 \mathrm{mg}$ aluminum hydroxide (Pierce Chemical Co., Rockford, IL, USA) with a total volume of $0.2 \mathrm{ml}$ on days 1,7 and 14 . From the day 15 to 22 , mice were treated with aerosolized OVA (5\% OVA) using ultrasonic nebulizer once daily consecutively. The mice were randomly divided into six groups: Control, OVA, DXM (dexamethasone), Low, Middle and High. Mice in the Low, Middle and High groups were respectively injected intravenously with $0.1,1$ and $10 \mathrm{mg} / \mathrm{kg}$ ALT III (Sigma-Aldrich, St. Louis, MO, USA) $1 \mathrm{~h}$ before OVA aerosol on days 15-22. At the same time, mice in the DXM group were treated with the equivalent DXM phosphate (Sigma, 10\% solution in PBS) by intragastric gavage. Mice in the Control group received the equivalent aluminum hydroxide via intraperitoneal injection and were exposed to a nebulized saline aerosol at the same time points as the OVA-challenged mice. At the end of the experiments, all animals were sacrificed by intraperitoneal injection with $200 \mathrm{mg} / \mathrm{kg}$ pentobarbital (Wuhan Boster Biological Technology, Ltd., Wuhan, China). The bronchoalveolar lavage fluid (BALF), blood samples and lung tissues were collected for subsequent analysis. Each experimental group included nine mice.

\section{Histopathology examinations}

Appropriate lungs tissues were conventionally fixed in 10\% formalin. In order to assess general morphology, paraffinembedded sections $(4 \mu \mathrm{m})$ were stained with hematoxylin and eosin (H\&E). Subsequently, all the slide sections were dehydrated with graded ethanol and xylene. The remaining paraffin-embedded lung tissues were cut into $2 \mu \mathrm{m}$ sections and stained with sirius red to assess pathological changes. A light microscope (Olympus Corp., Tokyo, Japan) was used for evaluating the pathological changes of lung tissues by an experienced pathologist unaware of the treatments.

\section{Measurement of cytokines production in BALF and OVA- specific immunoglobulin E (IgE) in serum}

The trachea was exposed by midline surgical section under anesthesia. Subsequently, $1 \mathrm{ml}$ cold PBS was used to lavage lungs and airways for three times. BALF (more than 90\%) was withdrawn and centrifuged (3000 rpm for $15 \mathrm{~min}$ at $4^{\circ} \mathrm{C}$ ) to obtain the supernatant. The concentrations of proinflammatory cytokines including IL-4, IL-5, IL-13 and antiinflammatory cytokines including IFN- $\gamma$, IL-10 and IL-12 in BALF as well as the concentration of OVA-specific IgE in 
serum were tested by enzyme-linked immunosorbent assay (ELISA) kits obtained from Shanghai Xitang Biotechnology Co., Ltd. (Shanghai, China) according to the instructions of the manufacturer.

\section{Analysis of inflammatory cells in BALF}

The collected BALF was centrifuged at $3000 \mathrm{rpm}$ for $15 \mathrm{~min}$ and the cell pellet was re-suspended in $100 \mu \mathrm{l}$ of PBS at $4^{\circ} \mathrm{C}$. The total number of cells was determined with a hemocytometer. The cell smears were prepared by cytocentrifugation (Cytospin 3; Thermo Scientific, Pittsburgh, PA, USA) and visualized using a Diff-Quik staining kit (Sysmex Co., Kobe, Japan). At least 200 cells per slide were counted under a light microscope, inflammatory cells including MAC, LYM, EOS and NEU were identified and counted according to the morphological criteria and staining features.

\section{Measurement of oxidative stress-associated factors}

The specimens of partial fresh lung tissues were collected from mice and immediately homogenized $(10 \%, w / v)$ in cold normal saline. The homogenate was centrifuged at 3000 $\mathrm{rpm}$ for $10 \mathrm{~min}$, followed by collection of the supernatant for assessment. ROS generation in homogenate was evaluated using the dichlorodihydrofluorescein diacetate (DCFH-DA) probe as described previously (Kalyanaraman et al. 2012). Additionally, the levels of malondialdehyde (MDA), lactate dehydrogenase (LDH), glutathione (GSH), superoxide dismutase (SOD) and catalase (CAT) were detected by the colorimetric method in accordance with the operating manual. Above mentioned assay kits were the products of Nanjing Jiancheng Bioengineering Institute (Nanjing, China).

\section{Immunohistochemistry assay}

The paraffin-embedded lung tissues were cut into $4-\mu \mathrm{m}$ thick sections, followed by deparaffinization and rehydration. These sections were incubated with $3 \% \mathrm{H}_{2} \mathrm{O}_{2}$ and then blocked with normal goat serum. Afterwards, the slides were incubated with primary antibodies targeting STAT3 (1:1000; Cell Signaling Technology; Boston, MA, USA). The sections were subsequently covered with a secondary peroxidase-conjugated antibody, followed by staining with diaminobenzidine staining (DAB; Beyotime, Shanghai, China) solution and counterstaining with hematoxylin. The slides were photographed under an optical microscope (Olympus Corp., Tokyo, Japan).

\section{Western blot analysis}

Total protein was isolated from the lung tissues on ice using radioimmunoprecipitation assay (RIPA) lysis buffer
(Beyotime, Shanghai, China). Protein concentration in each group was determined using a bicinchoninic acid (BCA) kit (Beyotime, Shanghai, China). Samples comprising $50 \mu \mathrm{g}$ of protein were electrophoresed in a denaturing $10 \%$ sodium dodecyl sulfate-polyacrylamide gel and then transferred to a polyvinylidene difluoride (PVDF) membrane. After soakage with 5\% skim milk, these membranes were incubated with primary antibodies at a 1/1000 dilution (Cell Signaling Technology; Boston, MA, USA). The bands were washed three times in Trisbuffered saline containing $0.1 \%$ Tween 20 (TBS-T) and then incubated with a 1/2000 dilution of HRP-conjugated anti-rabbit IgG (Cell Signaling Technology; Boston, MA, USA). Following incubation with secondary antibodies (Santa Cruz Biotechnology, CA, USA), the bands were visualized with an Odyssey Infrared Imaging Scanner (LICOR Biosciences). The intensity of bands was quantified by using Image-J software (NIH, MA, USA). GAPDH was used as an internal control.

\section{Statistical analysis}

Data were presented as mean \pm standard deviation (SD), and analyzed using Prism software (GraphPad Inc., San Diego, CA, USA). Student's $t$-test was used to compare the significant differences between every two groups. One-way analysis of variance (ANOVA) followed by Tukey's test was utilized to compare multiple groups. $p<0.05$ was considered statistically significant.

\section{Results}

ATL III treatment improved lung histological condition in asthma model mice

To assess the therapeutic effects of ATL III on OVA-induced lung injury, firstly the body weight and lung weight were determined, respectively. As presented in Figure 1A and B, OVA-stimulation dramatically decreased the body weight but increased the lung weight compared with the Control group. After treatment with ATL III, the body weight was significantly elevated in a dose-dependent manner, accompanied by a marked decline in lung weight. Subsequently, $\mathrm{H} \& \mathrm{E}$ and sirius red staining were executed for determining the pathological changes of lung tissues. As presented in Figure 1C, lung tissues in the Control group exhibited normal histology with no significant occurrence of inflammatory cells in peribronchial space. There was an increase in eosinophils infiltration in peribronchiolar and perivascular space and the erosion in peribronchiolar region in the lung tissues of OVA-sensitized and challenged mice compared with the Control group. 
A

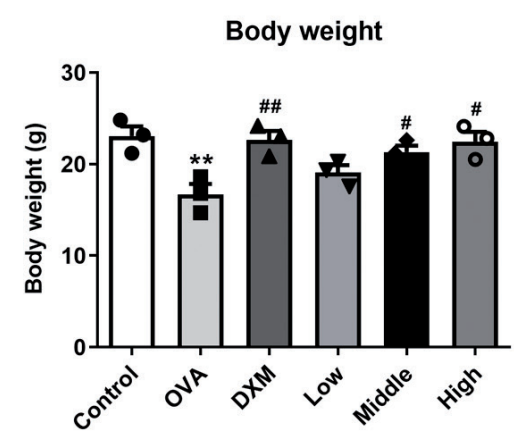

B

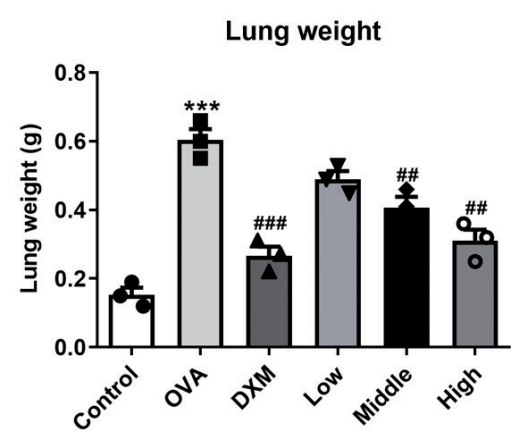

C

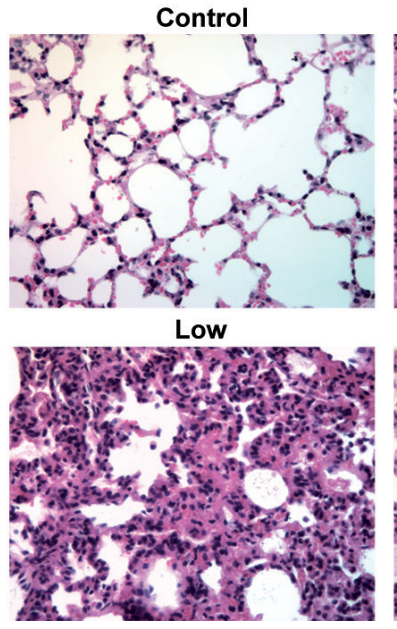

OVA

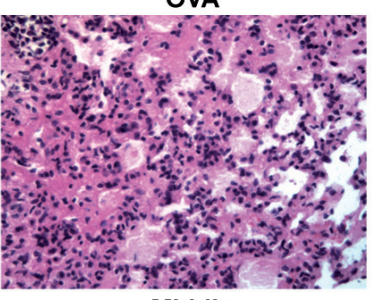

Middle

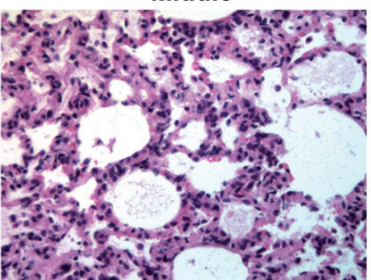

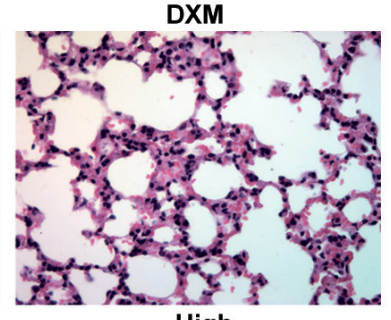
High

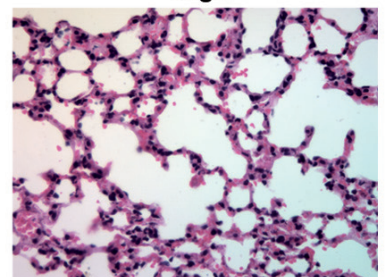

Figure 1. ATL III treatment significantly improved lung histological condition in asthma model mice. The body weight (A) and lung weight (B) of mice in each group were recorded. Mice in the Low, Middle and High groups were respectively injected intravenously with $0.1,1$ and $10 \mathrm{mg} / \mathrm{kg}$ ALT III. ${ }^{* *} p<0.01,{ }^{* * *} p<0.001 v s$. Control group; ${ }^{\#} p<0.05,{ }^{\# \#} p<0.01$, $\# \# p<0.001$ vs. OVA group. C. The changes of lung tissue pathobiology were determined using hematoxylin and eosin staining. ATL III, Atractylenolide III; DXM, dexamethasone; OVA, ovalbumin.
In addition, ATL III exhibited significantly reduced infiltration of inflammatory cells in a dose-dependent manner in contrast with the OVA group, representing the protective effect of ATL III. The high dose of ATL III presented the similar effects in the DXM group. Moreover, the sirius red staining was routinely used for determination of collagen deposition. Figure 2 shows that a large amount of collagen deposition appeared around the bronchioles after OVA challenge, while ATL III and DXM markedly inhibited this OVAinduced collagen deposition. These findings provided a clue that ATL III treatment significantly decreased peribronchial inflammatory cell infiltration and collagen deposition.
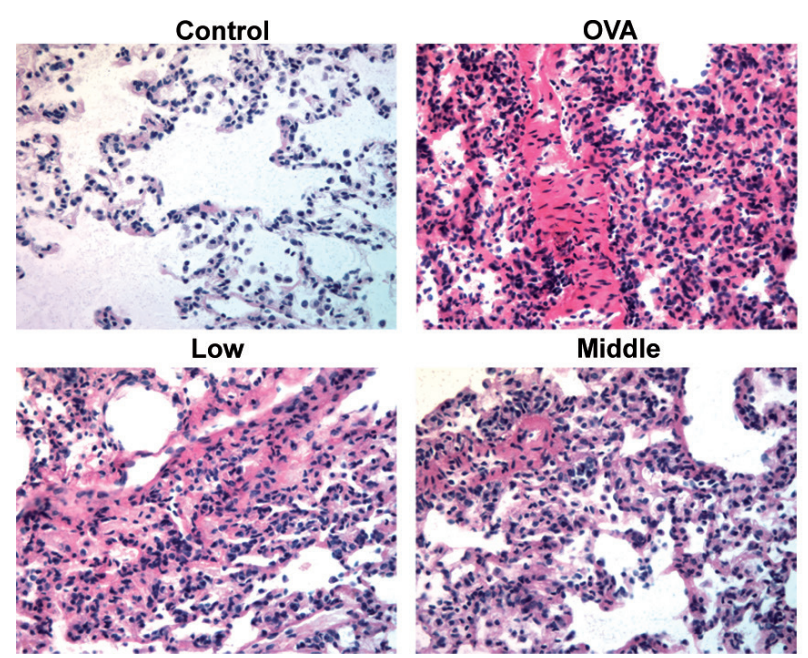

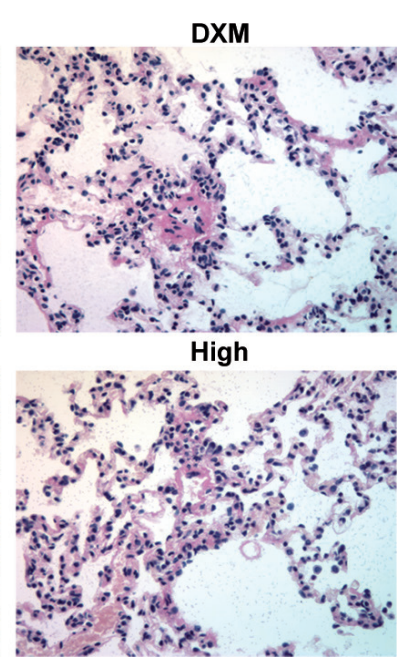

Figure 2. ATL III treatment notably improved lung histological condition in OVA-induced mice. Pathological changes of lung tissue were stained with sirius red staining. For abbreviations, see Fig. 1. 


\section{ATL III alleviated inflammatory responses in allergic asthmatic mice}

To study the effects of ATL III on inflammation induced by OVA, the levels of inflammation-associated cytokines were detected using ELISA kits. As observable from Figure 3, OVA stimulation led to great decrease in the contents of IFN- $\gamma$, IL-10 and IL-12 in BALF, which were key antiinflammatory cytokines during asthma. However, the levels of above-mentioned factors displayed significant increase in a dose-dependent manner after ATL III treatment. The DXM-treated group presented the same effects with the ATL III intervention groups. Additionally, a significantly decreased content of pro-inflammatory factors including IL-4, IL-5 and IL-13 in the BALF was observed compared to the OVA group, which were however not statistically different than the DXM group. These observations revealed that ATL III could balance the inflammatory cytokine level in the body, suggesting a beneficial effect of ATL III on OVAinduced inflammation.

\section{ATL III treatment changed the differential cells in BALF}

Subsequently, the number of MAC, LYM, EOS and NEU were identified and counted according to the morphological criteria and staining features. As shown in Figure 4, OVA sensitization and challenge noticeably elevated the total number of cells in the BALF with a prevalent increase in the levels of MAC, LYM, EOS and NEU in BALF compared with the Control group. However, treatment with ATL III notably reduced the total number of cells and above-mentioned cells in BALF in a dose-dependent manner. Consistently, DXM intervention displayed the same trends on the number of MAC, LYM, EOS and NEU as the ATL III-treated groups. The detailed phenotyping of immune cells reveals that the normal body immune response is not disturbed by ATL III treatment.

\section{ATL III inhibited the level of serum OVA-specific IgE in OVA-immunized asthmatic mice}

High levels of serum OVA-specific IgE antibodies are associated with the development of OVA-induced asthma model. Therefore, the level of OVA-specific IgE in serum, if measured, can be a marker for determining effective sensitization and treatments. In our study, OVA-specific IgE was not reported in control mice, whereas we observed a significant increase in serum OVA-specific IgE levels in OVA-immunized and challenged mice (Fig. 4). However, we did not notice any significant differences in the serum OVA-specific IgE level between ATL III- and DXM-treated groups. These data further signify the therapeutic role of ATL III in asthma management.

\section{ATL III attenuated oxidative stress in allergic asthmatic mice}

The effects of ATL III on oxidative stress were determined in allergic asthmatic mice. As presented in Figure 5, the levels of ROS, MDA and LDH were remarkably elevated in the OVA group compared to the Control group. ATL III treatment significantly reduced the levels of above-mentioned oxidative stress-related markers in a dose-dependently manner. Moreover, the content of GSH and the activities of SOD and

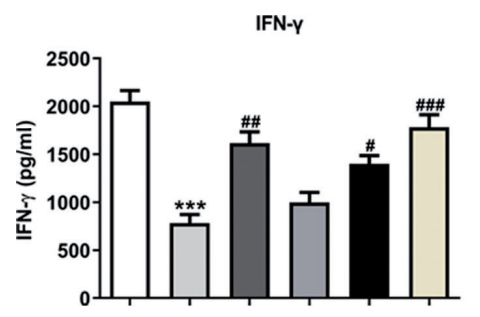

IL- 4

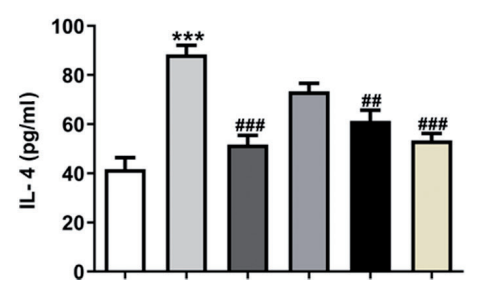

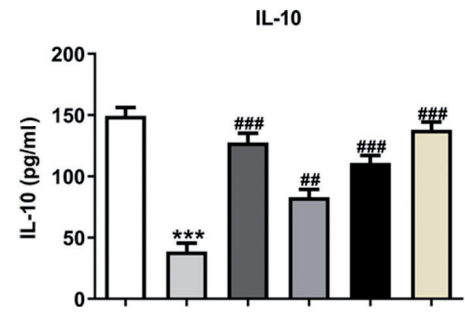

IL- 5

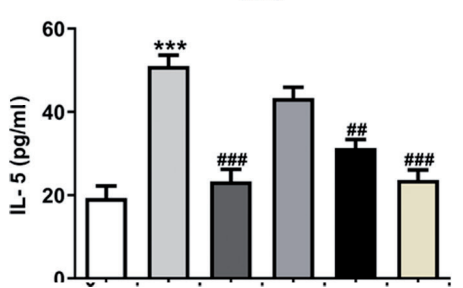

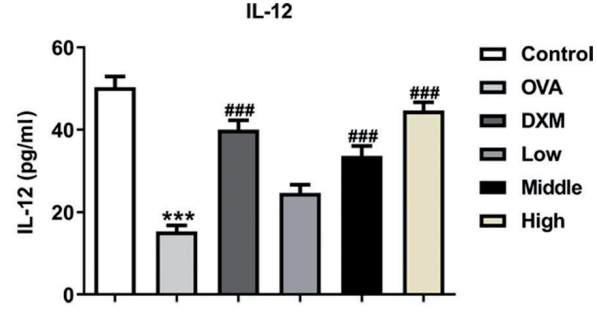

IL-13

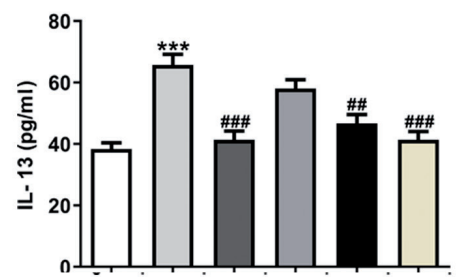

Figure 3. ATL III alleviated inflammatory responses in allergic asthmatic mice. The contents of IFN- $\gamma$, IL-10, IL-12, IL-4, IL-5 and IL-13 in BALF were detected using ELISA kits, respectively. ${ }^{* *} p<0.001$ vs. Control group; ${ }^{\#} p<0.05,{ }^{\# \#} p<0.01,{ }^{\# \# \#} p<0.001$ vs. OVA group. IFN- $\gamma$, interferon- $\gamma$; IL-10, interleukin-10; BALF, bronchoalveolar lavage fluid. For more abbreviations, see Fig. 1. 


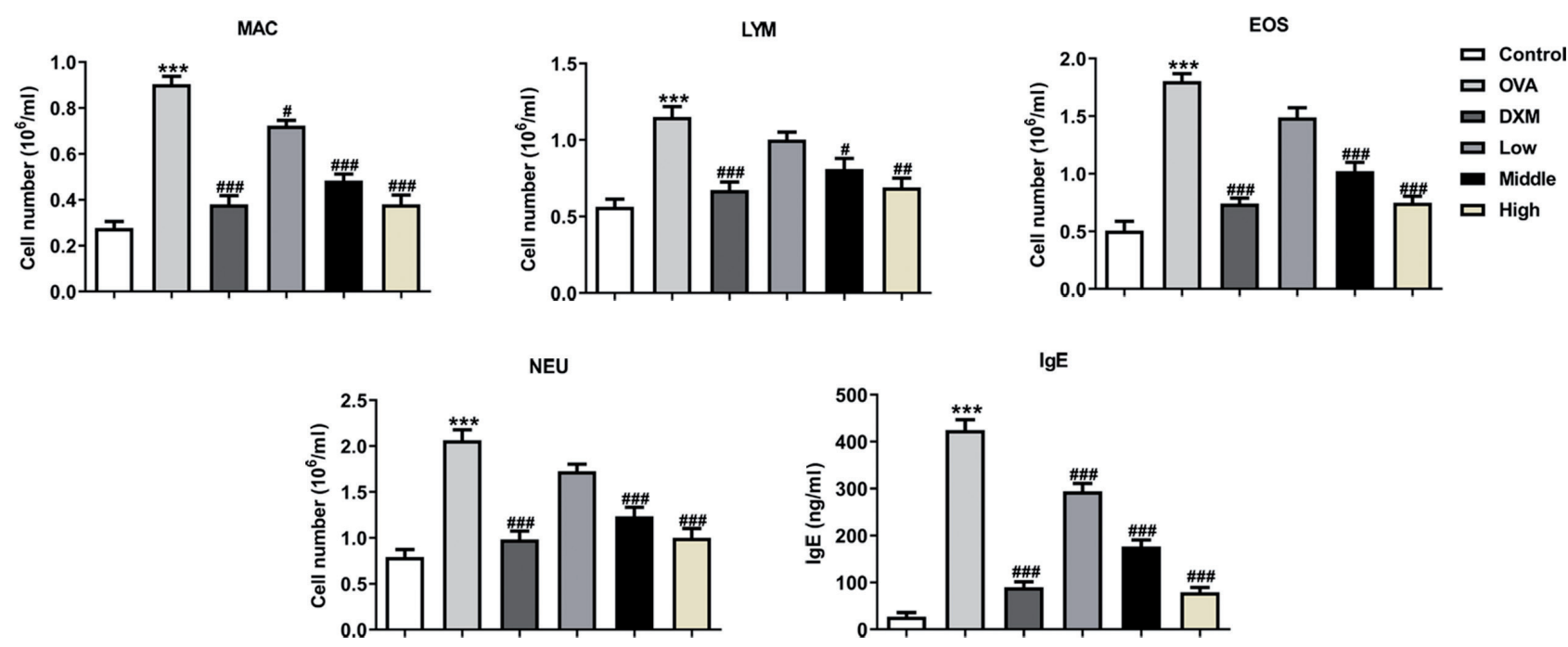

Figure 4. ATL III treatment changed the differential cells in BALF and the contents of OVA-specific IgE in OVA-immunized asthmatic mice. The number of MAC, LYM, EOS, NEU in BALF and the concentration of OVA-specific IgE in serum was evaluated. ${ }^{* *} p<0.001 v s$. Control group; ${ }^{\#} p<0.05,{ }^{\# \#} p<0.01,{ }^{\# \#} p<0.001$ vs. OVA group. MAC, macrophage; LYM, lymphocyte; EOS, eosinophil; NEU, neutrophil; BALF, bronchoalveolar lavage fluid. For more abbreviations, see Fig. 1.

CAT were notably decreased in the OVA-induced model mice compared with those in the Control group (Fig. 5). However, ATL III intervention exerted a marked restoration effect on the levels of GSH, SOD and CAT dose-dependently, which displayed the same effects with DXM. These findings indicated that ATL III attenuates oxidative stress in allergic asthmatic mice.
ATL III inhibited the expression of STAT3 in mouse airway tissues

To investigate the mechanisms of ATL III functioning in allergic asthmatic mice, the expression of STAT3 was examined using Western blot analysis. Results of Figure $6 \mathrm{~A}$ revealed that OVA stimulation significantly upregu-

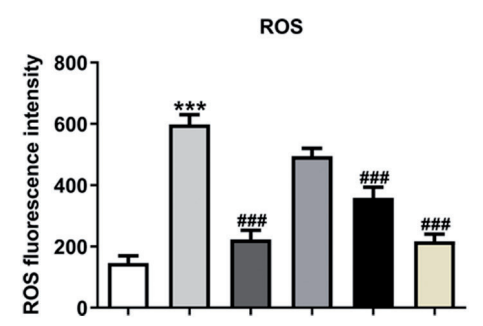

GSH

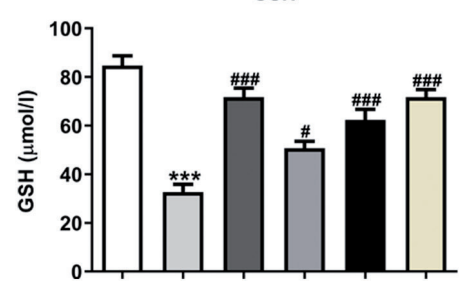

MDA

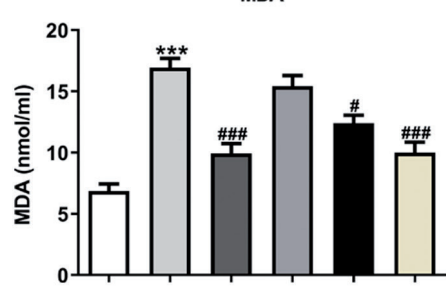

SOD

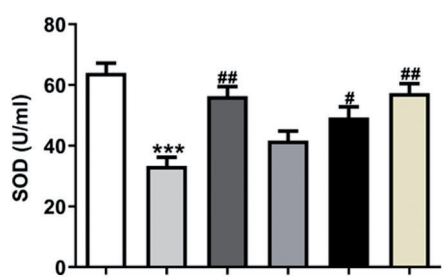

LDH

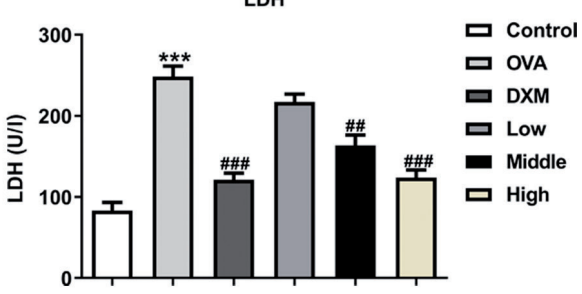

CAT

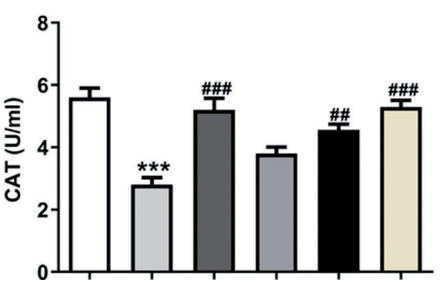

Figure 5. ATL III attenuated oxidative stress in OVA-induced mice. The levels of ROS, MDA, LDH, GSH, SOD and CAT were examined using commercially available kits. ${ }^{* * *} p<0.001$ vs. Control group; ${ }^{\#} p<0.05,{ }^{\# \#} p<0.01,{ }^{\# \# \#} p<0.001$ vs. OVA group. ROS, reactive oxygen species; MDA, malondialdehyde; LDH, lactate dehydrogenase; GSH, glutathione; SOD, superoxide dismutase; CAT, catalase. For more abbreviations, see Fig. 1. 
A

B
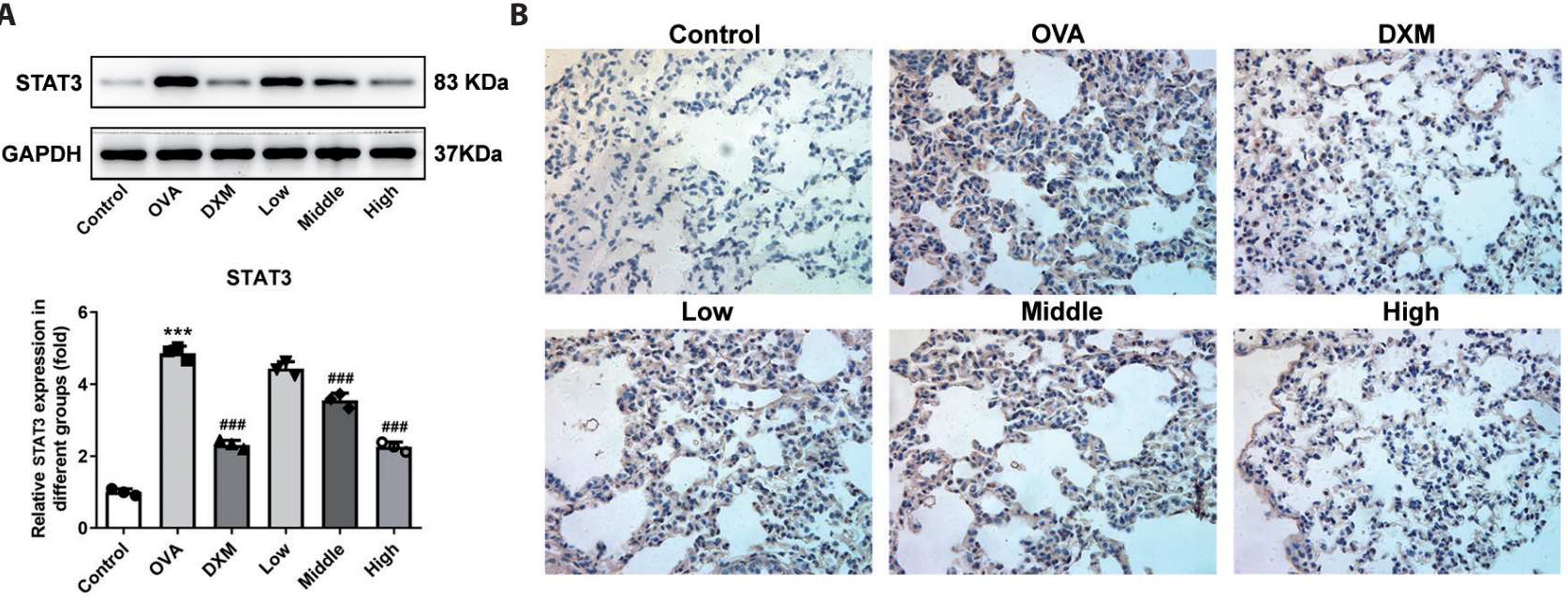

Figure 6. ATL III inhibited the expression of STAT3 in mouse airway tissues. The expression of STAT3 was detected using Western blot analysis (A) and immunohistochemistry assay (B). ${ }^{* *} p<0.001$ vs. Control group; ${ }^{\# \#} p<0.001$ vs. OVA group. STAT3, signal transducer and activator of transcription 3. For more abbreviations, see Fig. 1.

lated the expression of STAT3 compared with the untreated Control group, whilst ATL III treatment downregulated it in a dose-dependent manner without statistical difference when compared with the DXM group. And the results of immunohistochemistry assay exhibited in Figure 6B displayed the same variation as the Western blot analysis. These data proved our hypothesis that ATL III could suppress the STAT3 expression in OVA-induced mice model.

\section{Discussion}

Asthma is a chronic respiratory illness caused by inflammation and narrowing of tiny airways, with occasional or persistent symptoms, including shortness of breath, coughing, chest pain and wheezing (Wilson et al. 2012; Loftus and Wise 2016). The present study demonstrated that ATL III suppresses STAT3 expression and controls the inflammation and oxidative stress in OVA-induced mouse model.

Inflammation plays a key role in asthma, thus controlling the inflammation is the best strategy for treating asthma (Barnig et al. 2019). IL-4, IL-5 and IL-13 belong to Th2 cytokines, which contribute to the occurrence and progression of asthma (Hambly and Nair 2014; Parulekar et al. 2018). The role of IL-4 has been recognized in the development of allergic inflammation (Xia et al. 2018). Reports have demonstrated previously enhanced levels of IL-4 in BALF and increased expression of IL-4 mRNA in BAL cells in patients with allergic inflammation (Robinson et al. 1992, 1993; Walker et al. 1992). In the mouse models, IL-4 has been reported to be critical for the development of allergic airway inflammation (Brusselle et al. 1994, 1995).
Depletion of IL-4 or IL-13 using monoclonal antibodies is proven to be beneficial in asthma treatment (Hambly and Nair 2014). Moreover, mounting evidence has supported that IL-5 plays an important part in maturation and differentiation of EOS and B cells (Takatsu 2011). Differentiating B cells into plasma cells that secrete IgE is a complicated cascade of events in which cytokines are considered essential, suggesting the synthesis of IgE may rely largely on these cells (Yssel et al. 1998; Froidure et al. 2016; Matucci et al. 2018). IL-4 and IL-13 both induce the synthesis of IgE while IFN-gamma and IL-12 block it. Existing study has shown that ATL III decreased NLRP3 inflammasome activation and Th1/Th2 imbalances in models of asthma (Zhu et al. 2020). Our results showed that Th2 cytokines, including IL-4, IL-5 and IL-13, were conspicuously reduced after the treatment with ATL III. Similarly, ATL III markedly reduced the level of OVA-specific IgE in a dosedependent manner. Therefore, as a naturally occurring compound, ATL III targets the Th2 cytokines in a beneficial way, which represents a new therapeutic approach to the treatment of asthma.

A growing body of literature has shown that the imbalance between the reducing and oxidizing systems favor a more oxidative state present in asthma (Mishra et al. 2018). Endogenous and exogenous ROS, MDA, LDH and GSH play a significant role in the airway inflammation and are determinants of asthma severity (Erzurum 2016). MDA concentration is one of the most frequently used indicators of lipid peroxidation of the membranes that results from oxidative damage, suggesting an acute asthmatic attack (Ozaras et al. 2000). Given the present data, the concentration of MDA was remarkably elevated in the OVA group 
compared with the Control group, whereas ATL III-treated group showed a reduction in MDA level without being statistically different than the DXM group. Asthma is also associated with decreased antioxidant defenses, such as SOD and GSH (Comhair and Erzurum 2010). Imbalanced SOD and CAT antioxidant defense is a primary event in the pathophysiology of asthma (Comhair et al. 2005; Qu et al. 2017). Our results show that OVA sensitization diminished SOD and CAT activities in mice, whilst ATL III increased it in a dose-dependent manner. Through the above findings we proved that ATL III can balance the oxidative stress in asthmatic condition and reverse the signs and symptoms of asthma.

Previous studies have shown that STAT3 plays a role in the pathogenesis of asthma (Paul et al. 2009). STAT3 drives the development of Th17 cells and cytokine production by Th2 and Th17 cells, which contributes to asthma (Redhu et al. 2013). Notably, epithelial STAT3 was recognized as a critical regulator of allergen-induced inflammation in a mouse model of asthma (Borish 2016). Similarly, it has been reported that ATL III plays a significant role in the downregulation of STAT3 expression (Zhou et al. 2019). The present study proved that selective pulmonary epithelial STAT3 blocked by ATL III could prevent asthma in OVA-induced mouse model. Next, we examined the different cytokines driven by STAT3, which are responsible for the pathophysiology of asthma. In this study, DXM was used for the positive control group, which is considered to be a standard treatment for acute asthma (Cao et al. 2007; Shefrin and Goldman 2009). Our results showed that ATL III when administered in high dose, inhibited the STAT3 and showed no statistical difference than DXM group. Therefore, our results suggest a therapeutic role of ATL III in asthma management through inhibition of STAT3 expression.

\section{Conclusion}

To sum up, the present study demonstrated that ATL III suppresses STAT3 expression and controls the inflammation and oxidative stress in OVA-induced mouse model. Our finding provides a strong preclinical assessment based on the therapeutic role of ATL III in the management of allergic asthma. However, more studies on ATL III are needed and shall be beneficial to the demonstration of the duration of this effect.

Ethics approval and consent to participate. All experimental protocols were approved by the Ethics Committee on Animal Experiments of the First Affiliated Hospital of University of South China.

Conflict of interest. The author declares no conflict of interest.

\section{References}

Barnig C, Bezema T, Calder PC, Charloux A, Frossard N, Garssen J, Haworth O, Dilevskaya K, Levi-Schaffer F, Lonsdorfer E, et al. (2019): Activation of resolution pathways to prevent and fight chronic inflammation: Lessons from asthma and inflammatory bowel disease. Front. Immunol. 10, 1699 https://doi.org/10.3389/fimmu.2019.01699

Beharry S, Gidla D, Maharaj A, Bisram S, Sakhamuri S, Seemungal TA, Pinto Pereira LM (2015): Reality and understanding of asthma control. Chron. Respir. Dis. 12, 340-346 https://doi.org/10.1177/1479972315598692

Borish L (2016): The immunology of asthma: Asthma phenotypes and their implications for personalized treatment. Ann. Allergy Asthma Immunol. 117, 108-114 https://doi.org/10.1016/j.anai.2016.04.022

Brusselle G, Kips J, Joos G, Bluethmann H, Pauwels R (1995): Allergen-induced airway inflammation and bronchial responsiveness in wild-type and interleukin-4-deficient mice. Am. J. Respir. Cell Mol. Biol. 12, 254-259

https://doi.org/10.1165/ajrcmb.12.3.7873190

Brusselle GG, Kips JC, Tavernier JH, van der Heyden JG, Cuvelier CA, Pauwels RA, Bluethmann H (1994): Attenuation of allergic airway inflammation in IL-4 deficient mice. Clin. Exp. Allergy 24, 73-80

https://doi.org/10.1111/j.1365-2222.1994.tb00920.x

Cao D, Tal TL, Graves LM, Gilmour I, Linak W, Reed W, Bromberg PA, Samet JM (2007): Diesel exhaust particulate-induced activation of Stat 3 requires activities of EGFR and Src in airway epithelial cells. Am. J. Physiol. Lung Cell Mol. Physiol. 292, L422-429

https://doi.org/10.1152/ajplung.00204.2006

Comhair SA, Erzurum SC (2010): Redox control of asthma: molecular mechanisms and therapeutic opportunities. Antioxid. Redox Signal. 12, 93-124 https://doi.org/10.1089/ars.2008.2425

Comhair SA, Xu W, Ghosh S, Thunnissen FB, Almasan A, Calhoun WJ, Janocha AJ, Zheng L, Hazen SL, Erzurum SC (2005): Superoxide dismutase inactivation in pathophysiology of asthmatic airway remodeling and reactivity. Am. J. Pathol. 166, 663-674 https://doi.org/10.1016/S0002-9440(10)62288-2

Corrado A, Renda T, Polese G, Rossi A, Serena Aipo Study Group (2013): Assessment of asthma control: the SERENA study. Respir. Med. 107, 1659-1666

https://doi.org/10.1016/j.rmed.2013.08.019

de Groot LES, van der Veen TA, Martinez FO, Hamann J, Lutter R, Melgert BN (2019): Oxidative stress and macrophages: driving forces behind exacerbations of asthma and chronic obstructive pulmonary disease? Am. J. Physiol. Lung Cell Mol. Physiol. 316, L369-384

https://doi.org/10.1152/ajplung.00456.2018

Dogan MF, Parlar A, Cam SA, Tosun EM, Uysal F, Arslan SO (2020): Glabridin attenuates airway inflammation and hyperresponsiveness in a mice model of ovalbumin-induced asthma. Pulm. Pharmacol. Ther. 63, 101936 https://doi.org/10.1016/j.pupt.2020.101936

Edwan JH, Perry G, Talmadge JE, Agrawal DK (2004): Flt-3 ligand reverses late allergic response and airway hyper-responsiveness 
in a mouse model of allergic inflammation. J. Immunol. 172, 5016-5023

https://doi.org/10.4049/jimmunol.172.8.5016

Erzurum SC (2016): New insights in oxidant biology in asthma. Ann. Am. Thorac. Soc. 13 (Suppl. 1), S35-39

Foster PS, Mould AW, Yang M, Mackenzie J, Mattes J, Hogan SP, Mahalingam S, McKenzie AN, Rothenberg ME, Young IG, Matthaei KI (2001): Elemental signals regulating eosinophil accumulation in the lung. Immunol. Rev. 179, 173-181 https://doi.org/10.1034/j.1600-065X.2001.790117.x

Froidure A, Mouthuy J, Durham SR, Chanez P, Sibille Y, Pilette C (2016): Asthma phenotypes and IgE responses. Eur. Respir. J. 47, 304-319 https://doi.org/10.1183/13993003.01824-2014

Hambly N, Nair P (2014): Monoclonal antibodies for the treatment of refractory asthma. Curr. Opin. Pulm. Med. 20, 87-94 https://doi.org/10.1097/MCP.0000000000000007

Hodge DR, Hurt EM, Farrar WL (2005): The role of IL-6 and STAT3 in inflammation and cancer. Eur. J. Cancer. 41, 2502-2512 https://doi.org/10.1016/j.ejca.2005.08.016

Huang N, Liu K, Liu J, Gao X, Zeng Z, Zhang Y, Chen J (2018): Interleukin-37 alleviates airway inflammation and remodeling in asthma via inhibiting the activation of NF-kappaB and STAT3 signalings. Int. Immunopharmacol. 55, 198-204 https://doi.org/10.1016/j.intimp.2017.12.010

Ji GQ, Chen RQ, Wang L (2016): Anti-inflammatory activity of atractylenolide III through inhibition of nuclear factor- $\kappa B$ and mitogen-activated protein kinase pathways in mouse macrophages. Immunopharmacol. Immunotoxicol. 38, 98-102 https://doi.org/10.3109/08923973.2015.1122617

Ji NF, Xie YC, Zhang MS, Zhao X, Cheng H, Wang H, Yin KS (2014): Ligustrazine corrects Th1/Th2 and Treg/Th17 imbalance in a mouse asthma model. Int. Immunopharmacol. 21, 76-81 https://doi.org/10.1016/j.intimp.2014.04.015

Jones K (1989): Asthma--still a challenge for general practice. J. R. Coll. Gen. Pract. 39, 254-256

Kalyanaraman B, Darley-Usmar V, Davies KJ, Dennery PA, Forman HJ, Grisham MB, Mann GE, Moore K, Roberts LJ, Ischiropoulos $H$ (2012): Measuring reactive oxygen and nitrogen species with fluorescent probes: challenges and limitations. Free Radic. Biol. Med. 52, 1-6 https://doi.org/10.1016/j.freeradbiomed.2011.09.030

Kang TH, Han NR, Kim HM, Jeong HJ (2011): Blockade of IL-6 secretion pathway by the sesquiterpenoid atractylenolide III. J. Nat. Prod. 74, 223-227 https://doi.org/10.1021/np100686a

Li CQ, He LC, Jin JQ (2007): Atractylenolide I and atractylenolide III inhibit lipopolysaccharide-induced TNF-alpha and NO production in macrophages. Phytother. Res. 21, 347-353 https://doi.org/10.1002/ptr.2040

Liang P, Peng S, Zhang M, Ma Y, Zhen X, Li H (2017): Huai Qi Huang corrects the balance of Th1/Th2 and Treg/Th17 in an ovalbumin-induced asthma mouse model. Biosci. Rep. 37, BSR20171071 https://doi.org/10.1042/BSR20171071

Lim H, Cho M, Choi G, Na H, Chung Y (2015): Dynamic control of Th2 cell responses by STAT3 during allergic lung inflammation in mice. Int. Immunopharmacol. 28, 846-853 https://doi.org/10.1016/j.intimp.2015.03.051

Loftus PA, Wise SK (2015): Epidemiology and economic burden of asthma. Int. Forum Allergy Rhinol. 5 (Suppl. 1), S7-10 https://doi.org/10.1002/alr.21547

Loftus PA, Wise SK (2016): Epidemiology of asthma. Curr. Opin. Otolaryngol. Head Neck Surg. 24, 245-249 https://doi.org/10.1097/MOO.0000000000000262

Matucci A, Vultaggio A, Maggi E, Kasujee I (2018): Is IgE or eosinophils the key player in allergic asthma pathogenesis? Are we asking the right question? Respir. Res. 19, 113 https://doi.org/10.1186/s12931-018-0813-0

Mishra V, Banga J, Silveyra P (2018): Oxidative stress and cellular pathways of asthma and inflammation: Therapeutic strategies and pharmacological targets. Pharmacol. Ther. 181, 169-182 https://doi.org/10.1016/j.pharmthera.2017.08.011

Nikolskii A, Shilovskiy I, Andreev S, Kozhikhova K, Barvinskaia E, Vishniakova L, Babakhin A, Gaisina A, Kudlay DA, Khaitov M (2019): STAT3 gene suppression as an approach for the treatment of non-eosinophilic bronchial asthma. Allergy 74, OA0112

Ozaras R, Tahan V, Turkmen S, Talay F, Besirli K, Aydin S, Uzun $\mathrm{H}$, Cetinkaya A (2000): Changes in malondialdehyde levels in bronchoalveolar fluid and serum by the treatment of asthma with inhaled steroid and beta2-agonist. Respirology 5, 289-292 https://doi.org/10.1046/j.1440-1843.2000.00260.x

Parulekar AD, Kao CC, Diamant Z, Hanania NA (2018): Targeting the interleukin- 4 and interleukin-13 pathways in severe asthma: current knowledge and future needs. Curr. Opin. Pulm. Med. 24, 50-55 https://doi.org/10.1097/MCP.0000000000000436

Paul B, Mishra V, Chaudhury B, Awasthi A, Das AB, Saxena U, Saxena A, Chauhan LK, Kumar P, Raisuddin S (2009): Status of Stat 3 in an ovalbumin-induced mouse model of asthma: analysis of the role of Socs3 and IL-6. Int. Arch. Allergy Immunol. 148, 99-108 https://doi.org/10.1159/000155740

Qu J, Li Y, Zhong W, Gao P, Hu C (2017): Recent developments in the role of reactive oxygen species in allergic asthma. J. Thorac. Dis. 9, E32-43 https://doi.org/10.21037/jtd.2017.01.05

Redhu NS, Shan L, Al-Subait D, Ashdown HL, Movassagh H, Lamkhioued B, Gounni AS (2013): IgE induces proliferation in human airway smooth muscle cells: role of MAPK and STAT3 pathways. Allergy Asthma Clin. Immunol. 9, 41 https://doi.org/10.1186/1710-1492-9-41

Robinson DS, Hamid Q, Ying S, Tsicopoulos A, Barkans J, Bentley AM, Corrigan C, Durham SR, Kay AB (1992): Predominant TH2-like bronchoalveolar T-lymphocyte population in atopic asthma. N. Engl. J. Med. 326, 298-304 https://doi.org/10.1056/NEJM199201303260504

Robinson DS, Hamid Q, Jacobson M, Ying S, Kay AB, Durham SR (1993): Evidence for Th2-type T helper cell control of allergic disease in vivo. Springer Semin Immunopathol. 15, 17-27 https://doi.org/10.1007/BF00204623

Sarver N, Murphy K (2009): Management of asthma: new approaches to establishing control. J. Am. Acad. Nurse Pract. 21, 54-65 
https://doi.org/10.1111/j.1745-7599.2008.00375.x

Shefrin AE, Goldman RD (2009): Use of dexamethasone and prednisone in acute asthma exacerbations in pediatric patients. Can. Fam. Physician 55, 704-706

Simeone-Penney MC, Severgnini M, Tu P, Homer RJ, Mariani TJ, Cohn L, Simon AR (2007): Airway epithelial STAT3 is required for allergic inflammation in a murine model of asthma. J. Immunol. 178, 6191-6199 https://doi.org/10.4049/jimmunol.178.10.6191

Takatsu K (2011): Interleukin-5 and IL-5 receptor in health and diseases. Proc. Jpn. Acad. Ser. B Phys. Biol. Sci. 87, 463-485 https://doi.org/10.2183/pjab.87.463

Walker C, Bode E, Boer L, Hansel TT, Blaser K, Virchow JC (1992): Allergic and nonallergic asthmatics have distinct patterns of T-cell activation and cytokine production in peripheral blood and bronchoalveolar lavage. Am. Rev. Respir. Dis. 146, 109-115 https://doi.org/10.1164/ajrccm/146.1.109

Wilson SR, Rand CS, Cabana MD, Foggs MB, Halterman JS, Olson L, Vollmer WM, Wright RJ, Taggart V (2012): Asthma outcomes: quality of life. J. Allergy Clin. Immunol. 129, S88-123

https://doi.org/10.1016/j.jaci.2011.12.988

Xia F, Deng C, Jiang Y, Qu Y, Deng J, Cai Z, Ding Y, Guo Z, Wang J (2018): IL4 (interleukin 4) induces autophagy in B cells leading to exacerbated asthma. Autophagy 14, 450-464 https://doi.org/10.1080/15548627.2017.1421884
Yssel H, Abbal C, Pene J, Bousquet J (1998): The role of IgE in asthma. Clin. Exp. Allergy 28 (Suppl. 5), 104-109 https://doi.org/10.1046/j.1365-2222.1998.028s5104.x

Zhang NN, Park DK, Park HJ (2013): The inhibitory activity of atractylenolide capital SHA, Cyrillic, a sesquiterpenoid, on IgE-mediated mast cell activation and passive cutaneous anaphylaxis (PCA). J. Ethnopharmacol. 145, 278-285 https://doi.org/10.1016/j.jep.2012.11.004

Zhao H, Ji ZH, Liu C, Yu XY (2015): Neuroprotection and mechanisms of atractylenolide III in preventing learning and memory impairment induced by chronic high-dose homocysteine administration in rats. Neuroscience 290, 485-491 https://doi.org/10.1016/j.neuroscience.2015.01.060

Zhou K, Chen J, Wu J, Wu Q, Jia C, Xu YXZ, Chen L, Tu W, Yang G, Kong J, et al. (2019): Atractylenolide III ameliorates cerebral ischemic injury and neuroinflammation associated with inhibiting JAK2/STAT3/Drp1-dependent mitochondrial fission in microglia. Phytomedicine 59, 152922 https://doi.org/10.1016/j.phymed.2019.152922

Zhu C, Zhang L, Liu Z, Li C, Bai Y, Wang L (2020): Atractylenolide III reduces NLRP3 inflammasome activation and Th1/Th2 imbalances in both in vitro and in vivo models of asthma. Clin. Exp. Pharmacol. Physiol. 47, 1360-1367 https://doi.org/10.1111/1440-1681.13306

Received: October 15, 2020

Final version accepted: December 17, 2020 